\title{
Review on scanning pattern evaluation in laser-based additive manufacturing
}

\author{
Wei-Wei Liu $\odot,{ }^{a}$ Kazi Mojtaba Saleheen, ${ }^{\text {a,* }}$ Zijue Tang, ${ }^{a}$ Hao Wang, ${ }^{a}$ \\ Gamal Al-Hammadi, ${ }^{\text {a }}$ Ahmed Abdelrahman, ${ }^{a}$ Zhao Yongxin, \\ Shun-Gang Hua, ${ }^{a}$ and Feng-Tao Wang ${ }^{b}$ \\ ${ }^{a}$ Dalian University of Technology, School of Mechanical Engineering, Dalian, Liaoning, China \\ ${ }^{\mathrm{b}}$ Shantou University, College of Engineering, Shantou, Guangdong, China
}

\begin{abstract}
Laser-based additive manufacturing (LBAM) is a group of advanced manufacturing processes used to produce metal components and functionally graded products. Production in LBAM is either limited to the formation of thin or thick coatings on a substrate by laser metal deposition or the production of a fully functional metallic product by selective laser melting. In every case, LBAM fabricated components require optimization for the process parameters to avoid defects, such as porosity, crack holes, thermal deformation, and mechanical strength. As a key link in the laser additive manufacturing (LAM) process, laser scanning path planning is an effective strategy for balancing the temperature field of the formed part, avoiding stress concentration, and preventing deformation and cracking. Efficient, accurate, and reasonable planning of the laser scanning path is of great significance for improving the processing efficiency of the process data, prolonging the life of the laser scanning system, and improving the forming quality of the specimen. Through many studies, it was found that the scanning pattern of the lasers has a significant impact on the mechanical properties and deformations caused by a thermal mismatch during the process. Therefore, it is essential to have indepth knowledge about path planning in LBAM. Our review mainly focuses on the influence of scanning patterns on deformation, temperature, and mechanical properties in LBAM. Finally, our paper discusses the current study limitations and some future studies in LAM technology. (C) 2021 Society of Photo-Optical Instrumentation Engineers (SPIE) [DOI: 10.1117/1 .OE.60.7.070901]
\end{abstract}

Keywords: laser-based additive manufacturing; scanning pattern; process parameters; path planning.

Paper 20210270V received Mar. 12, 2021; accepted for publication Jul. 13, 2021; published online Jul. 27, 2021.

\section{Introduction}

Additive manufacturing (AM), also known as 3D printing, is a revolutionary method of manufacturing that allows for the development of lighter, more vital parts and systems. The best way to imagine any AM method is to consider the inverse of subtractive manufacturing processes such as milling. ${ }^{1}$ Subtractive manufacturing creates components by removing material, while AM creates structures by adding material layer by layer. Subtractive technologies have evolved dramatically over the last 20 years. The implementation of three-dimensional (3D) complex surface simulation software has replaced conventional code generation methods, such as $\mathrm{G}$ and $\mathrm{M}$ codes. $^{2}$ Additive and subtractive manufacturing are not mutually exclusive, despite some main differences. In reality, they are often used concurrently and at different stages of product creation and manufacturing. However, AM can be a viable alternative to conventional manufacturing processes. ${ }^{3}$ As a significant recent discovery, laser-based additive manufacturing (LBAM) has attracted widespread attention from scholars worldwide. After decades of development, the scope of laser applications has become more and more extensive. Nowadays, laser additive manufacturing (LAM) has been widely adopted for manufacturing various parts with complex

*Address all correspondence to Kazi Mojtaba Saleheen, kazi786@163.com 


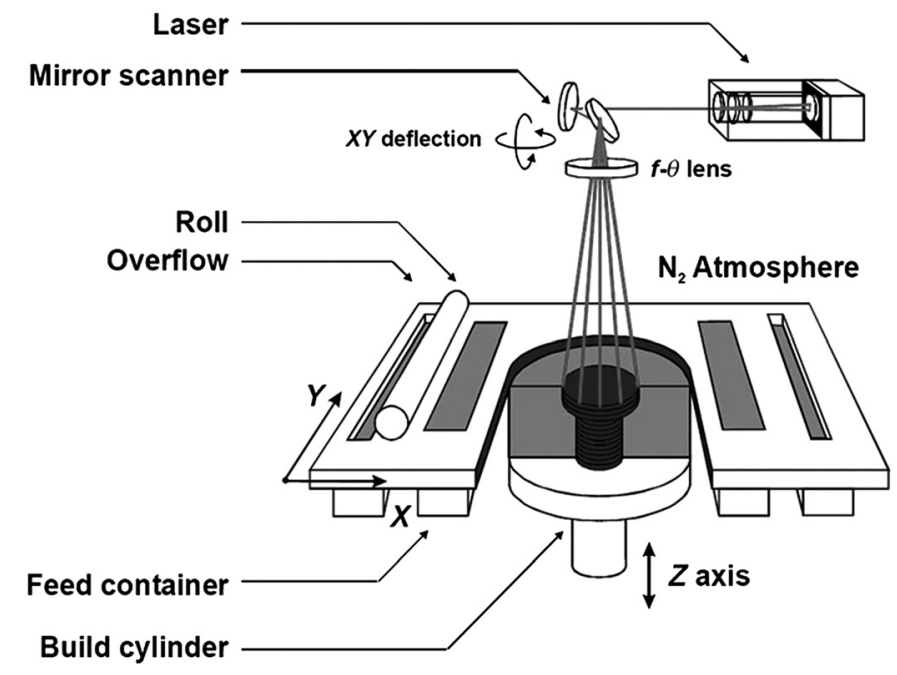

Fig. 1 A typical SLM machine layout. ${ }^{13}$

structures and outstanding performance ${ }^{4}$ that are used in tool-making, ${ }^{5}$ aviation and aerospace, ${ }^{6}$ and biomedical. ${ }^{7}$ Compared with the earlier traditional technologies, such as casting, forging, and extrusion, ${ }^{1}$ LAM technology has the following obvious advantages.

1. LAM can process functionally graded components by adjusting the chemical composition of the input metal material. ${ }^{8}$

2. The LAM technology forms parts using layer-by-layer material accumulation. The part structure is good optimization. They can be manufactured along the channel's contour, which improves the manufacturing precision. ${ }^{9}$

3. The laser has a high-power density, and the melting and cooling speeds are very fast during the process of making the material. The part's microstructure can be finely refined, which improves the comprehensive mechanical properties of the material. ${ }^{10}$

Laser-based powder bed fusion (PBF) and laser-based direct energy deposition (DED) are two different kinds of LAM. PBF utilizes the laser as a heat source to selectively fuse the powder layer by layer. Selective laser melting (SLM) is one of the PBF systems that is an AM process in which the components are created by consecutive deposition and melting of powder layers. ${ }^{11}$ SLM utilizes a comparatively high-power laser to fully fuse the powders compared with SLS and produce an utterly dense product. ${ }^{12}$ A typical SLM machine layout is shown in Fig. 1. The DED process utilizes a laser to fuse metal powders deposited by a feeder into the melt pool. ${ }^{14}$ The DED-AM machine is shown in Fig. 2. DED is also referred to as laser metal deposition (LMD), ${ }^{16}$ laser engineered net shaping (LENS), ${ }^{10}$ and laser cladding (LC). ${ }^{17}$ An argon jet is used in LMD to provide argon to the chamber, secure the laser optics, and shield the melt pool. The power supplied to the melt pool is aided by four argon jets placed around the main nozzle and directed at the melt pool, as shown in Fig. 3. LC technology, also known as laser deposition technology, is a kind of AM technology. This technology is based on the discrete + accumulation molding method; it breaks the traditional subtractive manufacturing processing method by combining surface repair technology and rapid prototyping manufacturing technology to achieve highprecision layered processing components. ${ }^{19}$

LAM also has some application problems. During the LAM process, the heating and cooling rates are very fast. Due to the difference in temperature gradient and thermal expansion coefficient in the LAM fabricated components, the temperature field distribution will be uneven and thermal stress will be generated. If not controlled and eliminated, it will lead to rupture. If the temperature field distribution of the part during the process can be known, the occurrence of layer cracking and matrix plastic deformation can be effectively avoided. Therefore, corresponding preventive measures to reduce cracks and deformation can be proposed. It is difficult to monitor the quality in real time in the LAM process due to the high-energy density laser 


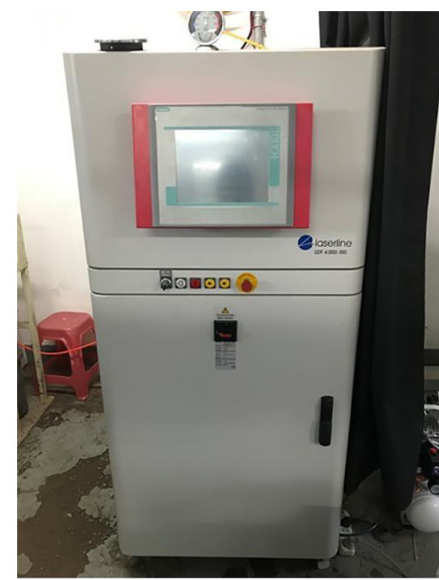

(a)

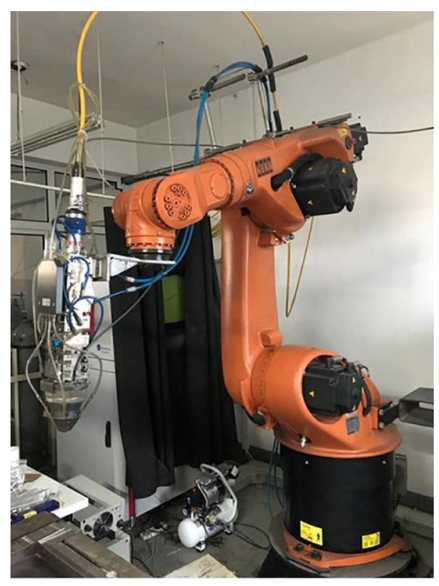

(b)

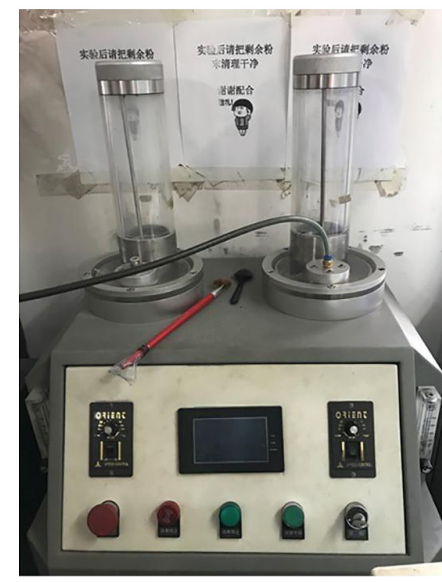

(c)

Fig. 2 DED-AM machine: (a) semi-conductor laser generator, (b) six-axis robot arm with a coaxial cladding head, and (c) powder feeder with carrying gas. ${ }^{15}$

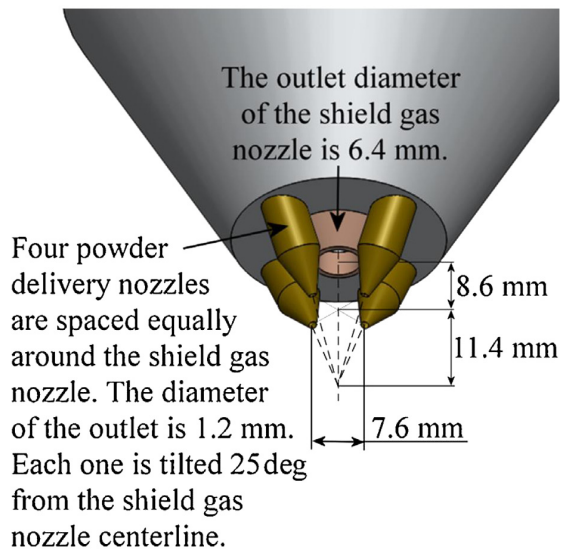

Fig. 3 An illustration of the LENS deposition head and powder nozzles. ${ }^{18}$

irradiation. People usually start analysis and research from the interaction between the laser and metal and the thermophysical and chemical phenomena. The temperature of the laser molten pool is very high. Measuring the temperature distribution of the molten pool experimentally is fairly challenging. In this prospect, numerical simulation has emerged. By establishing a numerical model on a computer that is very similar to the actual process, the computer numerical simulation can be used to predict the temperature field distribution of the LAM process. ${ }^{20-22}$

Thermal behavior is a crucial factor responsible for transforming microstructure, evolutions of residual stress, and part deformation significantly influencing th acquisition of high-quality products. To overcome these issues, many scholars have studied the scanning path planning of the laser in the LAM process. ${ }^{23-26}$ From the results analyzed by previous scholars, it was found that the scanning pattern has a significant effect on the temperature field, residual stress, thermal deformation, and mechanical properties of the LAM fabricated components. Therefore, it is essential to perform a scanning pattern evaluation in LAM to understand its influence. This paper focuses on the scanning pattern evaluation of LAM and considers its process signature that links the process parameters and product quality. This review highlights the following. Section 2 discusses the scanning patterns, Sec. 3.1 discusses the influence of scanning patterns on deformation, Sec. 3.2 describes the temperature field and thermal analysis, Sec. 3.3 explains the microstructure and mechanical properties, Sec. 4 discusses existing problems, and Sec. 5 concludes the paper. 


\section{Scanning Pattern}

In the LAM process, another critical issue is the generation and realization of the scanning path planning during the process. The scan path plays a vital role in the LAM process, so it has always been the focus and difficulty of research. Many researchers have carried out path studies and achieved a series of results, including various aspects of path planning. Effective deposition paths could offer the best inter-road and inter-layer connectivity, as is known to all, leading to enhanced shape and outstanding output of printed pieces, which is close to conventional processes due to a continuous polymer chain network. Therefore, selecting a suitable slicing method and proper filling patterns is very important. ${ }^{27}$ Optimizing the laser scan strategy could further minimize the effects of residual stress and reduce the specifications for the support structure without requiring adjustments to the SLM machine or the manufactured component design. ${ }^{25}$ The selection of a scanning strategy determines the morphology of the layer, which influences the layer's subsequent thickness, regularity, and continuity.

Optimal laser power, laser spot size, and scanning speed for various powder layer thicknesses must be used to generate continuous and stable single tracks. ${ }^{28-30} \mathrm{Jin}$ et al. ${ }^{31}$ presented a method of creating direction-parallel tool-paths for FDM. The suggested direction-parallel tool paths were found to bring substantial improvements in machining efficiency and surface quality. Boissier et al. $^{32}$ proposed a partial differential equation - ordinary differential equation optimal control method to optimize the trajectory based on existing patterns. The standard optimization problem was to meet the desired structure while avoiding overheating and preferably with the shortest path length possible. Their numerical outcomes contribute to a greater understanding of the problem and, as a result, short- and long-term solutions. Their findings also found that modifying a model and optimizing power and velocity can lead to more realistic results. Carraturo et al. ${ }^{33}$ used finiteelement (FE) analysis to examine residual stresses for the nine different laser scan control strategies to identify the strategy that minimizes residual stress magnitude. The findings demonstrate that a constant power density scan approach tends to be the most efficient at reducing residual stresses in the domain under consideration. Zhang et al. ${ }^{34}$ adopted a sub-sector scan mode for (SLM) technology to optimize the output of nearly completely dense metal parts. This scan pattern helps raise the temperature more efficiently in small sub-sectors to melt the metal powder. Jhabvala et al. ${ }^{35}$ analyzed different potential scanning strategies, and those that lead to homogeneous component heating up to its melting point were identified. Son et al. ${ }^{24}$ proposed a new technique for scanning a complex surface model with several patches. The established method calculates the number of scans, scan orientation, and scan line. Shishi et al. ${ }^{36}$ adopted two different scanning strategies to prepare a scanning path. The findings suggest that selecting an effective scanning strategy will minimize tensile stress and increase the mechanical properties of the components. Zhao et al. ${ }^{37}$ proposed an algorithm for rapid prototyping, a layered manufacturing technology, to develop an efficient scanning route. The results showed that the proposed algorithm enhances the machine's equality and advances the machine's reliability. Given that error situations occurred in a single laser scanning path, the optimal scanning path was created. The developed method allows the desired laser spot size to scan a wider planar surface. ${ }^{38}$

\section{Influence of Scanning Pattern on Deformation, Temperature Field, and Mechanical Properties in LBAM}

\subsection{Deformation}

The fundamental cause of LAM-induced distortion is the nonuniform heating and cooling of material during the LAM process, which produces elastic and plastic strains due to the mismatch of thermal expansions and constrictions between the melt pool zone and the surrounding zone in the parts. The influence of different laser scanning patterns on the deformation of the substrate is studied at this angle to reduce the deformation of the LAM fabricated parts and reduce the workload. A laser/powder deposition method was applied to the Shape Deposition Manufacturing System at Stanford University. The results show that both selective deposition and the use of a low CTE material such as INVART can significantly reduce the deformation of simple beams due to internal stresses. ${ }^{39}$ Gao et al. ${ }^{40}$ examined the impact of the deposition pattern 
on the substrate's deformation during direct laser fabrication. Eight laser scanning patterns were designed to create a cylinder on an asymmetrical IN718 arc substrate, respectively. The findings indicate that the substrate deformation along the $z$ direction for all scanning patterns is greater than the two other directions. Residual stress-induced warping is one of the most pressing concerns in the direct deposition of fully dense metals, leading to undesirable losses in dimensional resistance. ${ }^{41-43}$ Biegler et al ${ }^{44,45}$ introduced a novel method for measuring distortions experimentally and using the effects to validate numerical simulations. In conjunction with optical filters, in-situ distortions and periodic expansion and shrinkage are calculated by digital image correlation. When the experimental and numerical results are compared, the length direction of the sample has a strong agreement, while the height direction has quantitative deviations. Nie et al. ${ }^{46}$ performed a numerical simulation of the laser hot-wire AM to obtain the temperature, stress, strain fields, and distortion. The findings demonstrate that temperature fluctuates consistently with layer-by-layer deposition and that the overall distortion increases steadily during deposition. An accurate thermal-mechanical model was developed to propose controlling distortion in a wire-fed electron-beam thin-walled Ti-6Al-4V freeform. The overall distortion along the $x$ axis was reduced to $0.12 \mathrm{~mm}$ using a current dynamic scheme. The distortions along the $x$ and $z$ axes were reduced to almost zero using a constant temperature limit at the bottom of the substrate. ${ }^{47}$ Chen et al. ${ }^{48}$ proposed a multiscale process simulation framework for effectively and reliably simulating residual distortion and stress at the part scale. The effectiveness of this proposed framework is shown by simulating a double cantilever beam and a canonical component of different wall thicknesses and comparing the results with experimental measurements, which shows excellent consensus. Ramos et al. ${ }^{26}$ presented a novel scanning strategy implemented in the SLM manufacturing technology to reduce the residual stress produced during the buildup process. The scanning strategy implemented appeared to be the most effective method for reducing the component's deformation. Foteinopoulos et al. ${ }^{49}$ established a 3D thermal simulation method for the PBF process. The proposed method provides satisfactory and computationally low-cost results in terms of the strength of predicted thermal stresses and deformations. A new distortion control approach was developed and validated experimentally, based on optical 3D scan measurements. ${ }^{50}$ The findings of the study revealed that distortion reduction in SLM is now applicable on industrial macro-scale parts. The produced distortion in SLM was compensated for by changing the original geometry with $\mathrm{FE}$ expected distortion.

Moreover, many scholars have studied the influence of laser scanning patterns on residual thermal stresses and distortion using FE analysis. It was shown that choosing a proper scanning pattern can significantly reduce the material deformation and residual stress and can enhance the model accuracy. ${ }^{11,20,51-58}$ Figure 4 shows the LSFed parts' distortions using the raster, offset-in, offset-out, and fractal deposition patterns. Concerning as-built component warping and porosity, a newly established scan strategy is compared experimentally with its commercial equivalent. A significant decrease in warping and porosity resulted from the new approach. ${ }^{59} \mathrm{Li}$ et al.$^{60}$ created an FE model for quick prediction of SLM fabricated components distortion using four laser scanning strategies. Results showed that the horizontal scanning pattern produced the slightest longitudinal deflection. In contrast, the crosswise direction produced the biggest deflection. The DED-induced distortions and residual stresses were analyzed using a 3D thermo-mechanical FE model. ${ }^{61}$ It was found that different scanning techniques used during the preheating process significantly impacted distortion. Some modifications of the actual scanning sequence have been proposed to reduce the computational expense of the simulation framework without compromising quality. Klingbeil et al. ${ }^{62}$ experimented on plate-shaped specimens provided by two methods of direct metal deposition. The experimental findings indicated that both the material deposition process and the deposition direction may significantly influence subsequent warping. Nickel ${ }^{63}$ explored how the pattern used to deposit a layer affects the substrate's warpage and analyzed the inter-layer surface defect known as the Christmas Tree Step. Their findings revealed that bolting the substrate down during deposition has a major impact on a component deflection for the elastic-perfectly plastic model. It is necessary to understand how distortion accumulates during AM in a machined surface to design techniques to reduce stress and distortion. Denlinger et al ${ }^{64}$ performed a distortion measurement on titanium and nickel alloy with different interlayer dwell times. This study's findings showed that the addition of dwelling time during the deposition process allows for additional cooling, which results in lower distortion and residual 

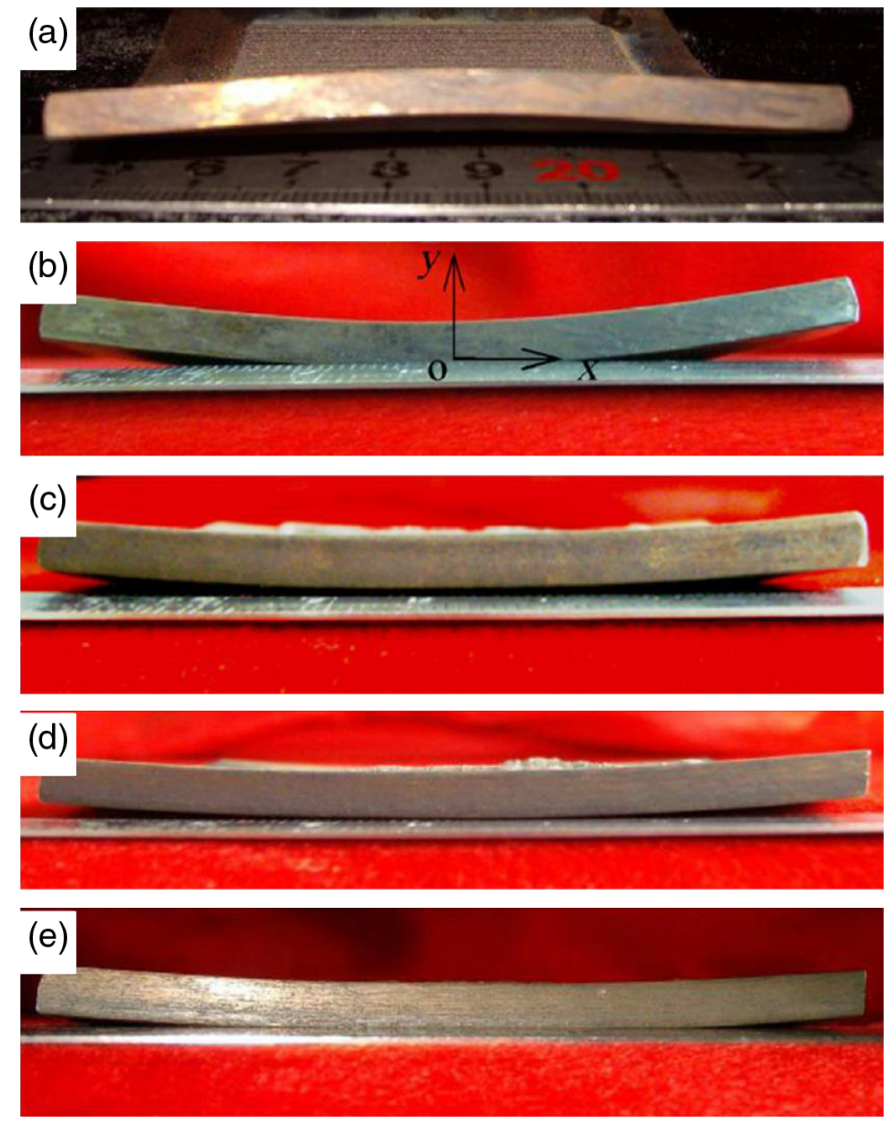

Fig. 4 Exhibitions of LSFed part deformation induced by different deposition patterns (a) raster parallel to the $z$ direction, (b) raster parallel to the $x$ direction, (c) offset-in, (d) offset-out, and (e) fractal. $^{51}$

stress. Kruth et al. ${ }^{65}$ illustrated the benefit of the processing parameters by SLM to obtain maximum density metal parts. Proper scanning patterns have greatly minimized thermal deformations and the beneficial effect of vaporization to limit the balling effect. Qian et al. ${ }^{66}$ developed a helix scan strategy and progressive scan strategy for SLM technology to reduce the deformation of manufactured layers. From his research, it was found that the helix scan strategy minimizes the deformation of the manufactured layers.

\subsection{Temperature Field and Thermal Analysis}

The LAM process involves complex physical and chemical reactions, such as microstructure, changes in segregation, thermophysical properties, rapid melting and cooling, and many other processes. The uneven distribution of the substrate's temperature field during the laser irradiation causes the cladding part's deformation. The temperature field should be distributed evenly to solve this issue during the process. Under the current engineering application technology conditions, it is challenging to use experimental methods to measure heat transfer and temperature field distribution in the molten pool. In comparison, the numerical simulation of the temperature field, establishing a model, and determining boundary conditions using a computer can obtain more accurate thermophysical parameters, mass and heat flow fields, as well as other important information. The numerical analysis of the temperature field is based on the actual situation of the process. Figure 5 exhibits the temperature field contours for different laser scanning patterns.

The non-uniform distribution of temperature and the rapid thermal cycle can lead to thermal distortion and dimensional errors in the LC process. ${ }^{68,69}$ So, understanding and monitoring thermal behavior during the LAM process is essential. ${ }^{70}$ Gong et al. ${ }^{71}$ analyzed the planar LC temperature field with a length direction, a width direction, and a simulated helical scanning path. 


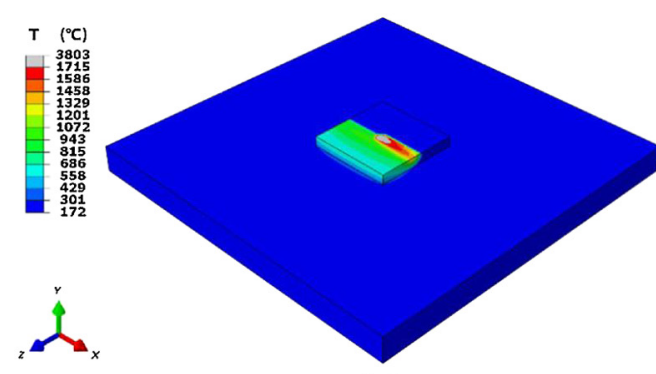

(a)

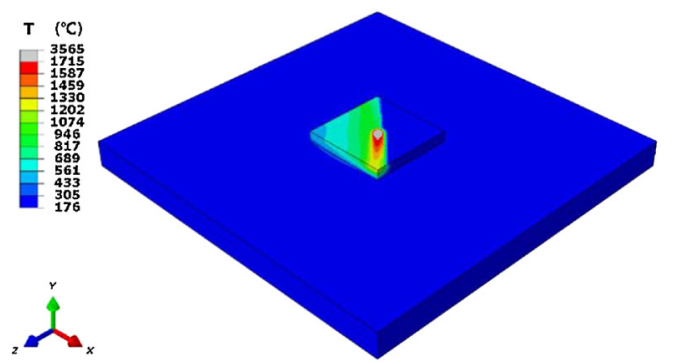

(b)

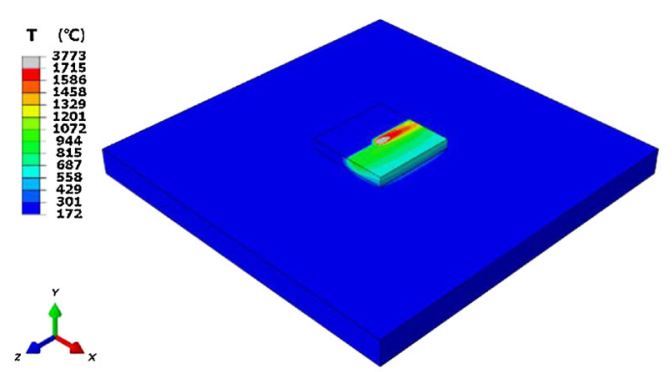

(c)

Fig. 5 Temperature field distribution plot for three scanning strategies: (a) line scan, (b) 15-deg rotate scan, and (c) 90-deg rotate scan. ${ }^{67}$

Results showed that the laser scanning path could significantly affect temperature distribution. Foroozmehr and Kovacevic ${ }^{72}$ and Asikainen et al. ${ }^{73}$ established a model for evaluating the influence of the scanning pattern on the final distribution of stress, and their findings revealed that the scanning pattern could alter the process temperature history, and as a result, the final properties of the deposited material may change. Ghosh and $\mathrm{Choi}^{74}$ analyzed the influence of deposition patterns on induced thermal stresses. It was found that choosing a proper deposition pattern can reduce thermal mismatch, resulting in less warping. From the consistency of the layer viewpoint, the analysis of temperature distribution within the metallic layer during metal laser sintering is essential. ${ }^{75-81}$ Denlinger and Michaleris ${ }^{82}$ proposed an approach for modeling stress relief effects at high temperatures during laser DED processes with various inter-layer dwell periods. In their findings, the thermal behavior of workpieces is found to be highly reliant on dwell time. In the powder layer, the high temperature produced results in significant distortion of the component and caused thermal and residual stresses. Song et al. ${ }^{67}$ proposed a combination of FE simulation and experimental verification to analyze the influence of scanning strategies on thermal behavior and residual stress distribution. The temperature distribution simulation results show that scanning strategies significantly influence the temperature field. Patil et al. ${ }^{83}$ conducted a numerical experiment to investigate the scan pattern's impact and the time needed to cool down the powder around the melt pool. His research explores the significance of hatch patterns for the thermal history of successive subdomains. The FE formulation for heat transfer in a material with isotropic thermal properties has the following governing equation:

$$
-\left(\frac{\partial q_{x}}{\partial x}+\frac{\partial q_{y}}{\partial y}+\frac{\partial q_{z}}{\partial z}\right)+Q=\rho c \frac{\partial T}{\partial t}
$$

where $q_{x}, q_{y}, q_{z}$ are components of heat flow through a unit area. According to Fourier's law,

$$
q_{x}=-k \frac{\partial T}{\partial x} \quad q_{y}=-k \frac{\partial T}{\partial y} \quad q_{z}=-k \frac{\partial T}{\partial z}
$$

where $K$ is the thermal conductivity coefficient of media, $Q$ is the $Q(x, y, x)$ inner heat generation rate per unit volume, $\rho$ is the material density, and $c$ is the heat capacity. 
A coupled thermo-mechanical simulation was developed for two different scan strategies to predict residual stress. The findings showed that the scan vector length directly impacts temperature oscillations. Due to the shorter time between neighboring scans, the unidirectional approach has a more consistent minimum temperature. ${ }^{25}$ An experimentally determined surface convection was integrated into a DED AM thermo-mechanical model. ${ }^{18}$ The residual stress measurements and in-situ deflections suggest that the measurement-based convection model generates more precise stress measurements in all situations. Dai and Shaw ${ }^{84}$ performed a 3D simulation of finite elements to investigate the temperature field using a moving laser beam to process several material components. The distribution of temperature, transient stress, residual stress, and distortion of a multi-material part have significant effects on laser processing conditions and material properties, especially thermal conductivity and thermal expansion. The numerical simulation was carried out using the ANSYS code ${ }^{85,86}$ Bian et al ${ }^{87}$ investigate the effect of laser power and two scanning techniques (stripe scanning and chessboard scanning) by SLM on the residual stress distribution in $316 \mathrm{~L}$ steel. The implementation of stripe scanning (rather than chessboard scanning) and an increase in laser power from 160 to $200 \mathrm{~W}$ typically increased tensile residual stress in the region of interest. Furthermore, compared with switching between two scanning methods, the increase in laser power from 160 to $200 \mathrm{~W}$ tended to have a more significant effect. Many attempts have been made to develop the LC process from the 1D model to the $2 \mathrm{D}$ model, including thermal and structural analysis ${ }^{88,89}$ The multilayer problem modeling is equally important because the thermal correlations of subsequent layers affect the temperature gradients that regulate heat transfer and thermal stress growth. An advanced simulation technique known as element birth and death is used in modeling the 3D temperature field in several layers in a powder bed. ${ }^{90}$ The results demonstrated that heated regions are subjected to rapid thermal cycles correlated with corresponding thermal stress cycles. The variation in heat dissipation at different substrate locations was investigated to prevent defects such as overburning and collapsing at the LC layer's boundary. ${ }^{91}$ Over-burning and collapsing regions are predicted and experimentally validated for different direction cladding and the same side cladding. The findings show that the scanning path has a significant impact on boundary over-burning and collapsing. The cladding consistency of the boundary can be improved with the same direction cladding and different side cladding without affecting machining performance, material utilization, or process parameter rationality.

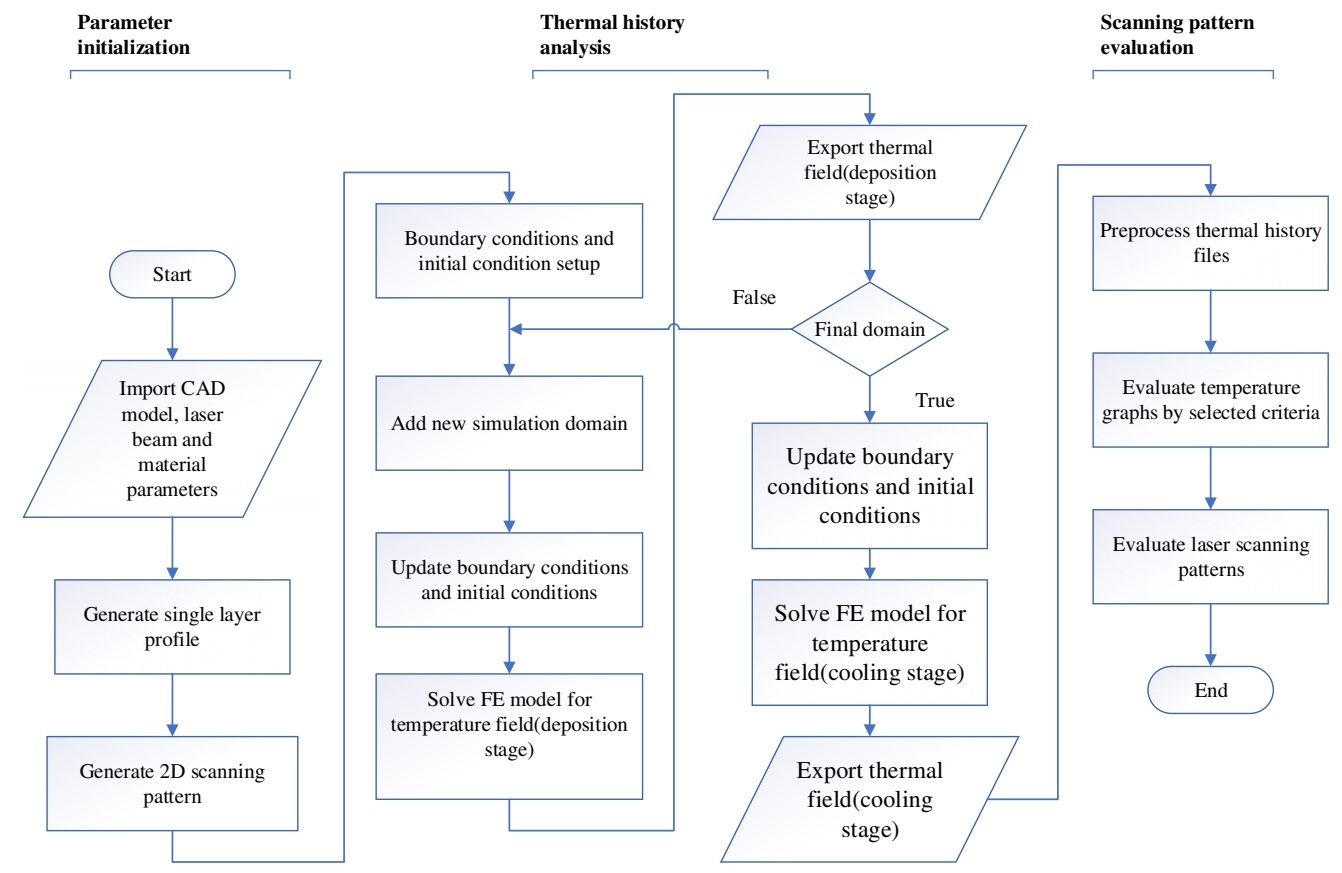

Fig. 6 Scanning pattern evaluation framework scheme. ${ }^{95}$ 
A 3D transient heat transfer model was used to reliably measure the transient temperature field for residual stress and distortion simulation. ${ }^{92}$ The results demonstrated that decreasing the layer thickness can significantly reduce the residual stress. Alimardani et al. ${ }^{93}$ proposed a model to analyze the influence of the workpiece being preheated and clamped to the positioning table. Results showed that preheating enhanced efficiency by eliminating both the thermal stresses and the settling time in the first layer to create a stable melt pool. Ma and $\mathrm{Bin}^{94}$ Ren et al.$^{95,96}$ investigated the impact of laser scanning patterns on temperature, residual thermal stresses, and distortion in LAM. An effective 3D thermal history evaluation FE framework was designed to predict temperature field evolution for arbitrary scanning patterns. The scanning patterns evaluation framework used to predict the thermal history is shown in Fig. 6.

In laser heating, the heat transfer process is a very complex issue. During the laser heating process, the energy source comes primarily from laser beam radiation, latent phase change heat, and deformation heat. Latent heat is released when the metal content is melted or solidified, which will affect the heat transfer process. Furthermore, the material's plastic deformation can produce deformation heat, which is typically very low compared with the other two heat sources. ${ }^{97}$

\subsection{Microstructure and Mechanical Properties}

Many studies about LAM in manufacturing structures or components using different materials, such as stainless steel, titanium alloys, aluminum alloys, and nickel-base superalloys, have been published. The mentioned studies have concerned the investigations of the metal workpieces' microstructures and mechanical properties or samples fabricated by LAM. For AM, microstructures and mechanical properties are key factors studied to monitor and enhance the consistency of the final product. ${ }^{98-102}$ Rare data about the influence of laser beam scanning paths on the material's microstructures and mechanical properties exists in literature until now. Many scholars have studied the impact of scanning patterns on microstructure and mechanical properties in LBAM; their findings clearly state that the scanning pattern has a significant effect on microstructure and mechanical properties of LBAM fabricated components. ${ }^{15,103-107}$ So, to control its microstructures and mechanical properties, it is essential to study the differences in structures and properties of LSF structures or components deposited with different laser beam scanning paths. Figure 7 shows the microstructures of LSF Inconel 718 samples after heat treatment with varying directions of scanning. The distribution of recrystallized grain sizes reflects the allocation of residual thermal stress. A previous study ${ }^{108}$ proved that columnar dendrites expand epitaxially along the deposition direction, turning to distribute equiaxed grains after hightemperature recrystallization unevenly. A more uniform allocated grain size in the samples indicates that its residual thermal stress distribution is more consistent. ${ }^{109}$ Specific treatments are required to obtain optimal mechanical properties due to the particular microstructure resulting from the SLM method. Different microstructural characteristics in ferritic and austenitic steel DED FGM sections were examined. ${ }^{110}$ The epitaxial grain growth along the building direction creates an equiaxed grain structure in both parts. In the bidirectional scan, the ferritic steel
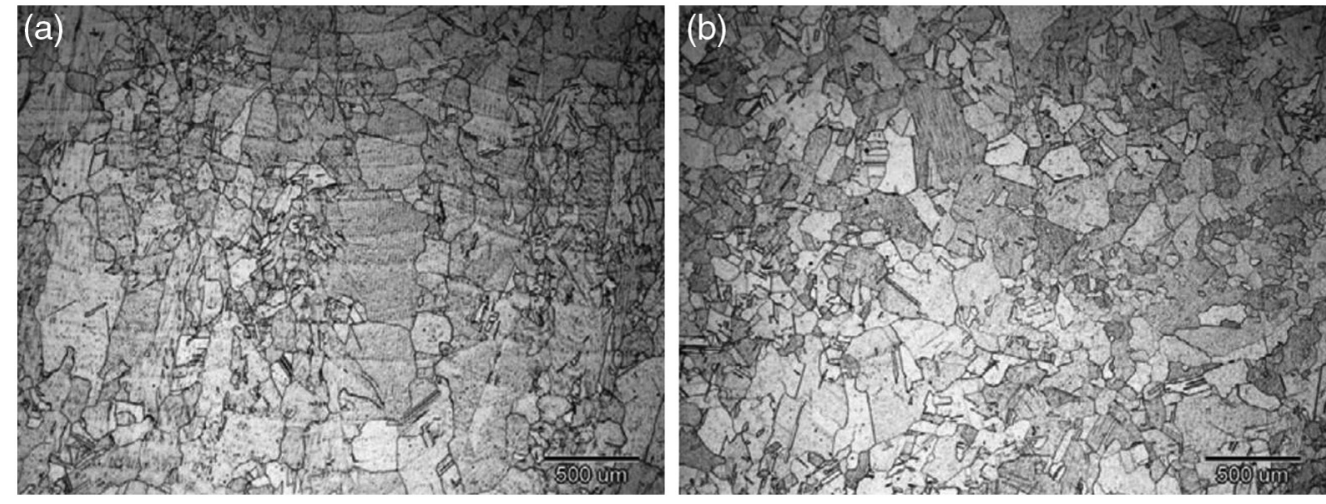

Fig. 7 Recrystallized microstructures of LSF Inconel 718 alloy. (a) Deposited with single direction raster scanning and (b) deposited with cross direction raster scanning. ${ }^{103}$ 
component has a preferentially inclined grain structure. At the same time, it becomes less favored in orthogonal and island scans due to the combination of scanning strategies between interlayers. Ali et al. ${ }^{111}$ investigated the impact of scanning and rescanning strategies on residual stress formation and mechanical properties of SLM fabricated parts. It was found that the 90-deg alternating scanning strategy produced the least amount of residual stress. Still, there was no apparent connection between mechanical properties. Since the temperature distribution and cooling rate determine the sintered material's properties, numerical simulations allow SLM to estimate the optimal conditions for producing objects with the preferred microstructure and mechanical properties. ${ }^{112}$ Sheng et al. ${ }^{113}$ established a 3D multitrack and multilayer thin-wall to enhance the quality of thin-wall metal parts provided by the direct laser deposition shaping process using the element birth and death method.

Based on the study of mechanical components' characteristics, appropriate scanning route planning impacts the cracking failure behavior of thin-wall metal components. Kruth et al. ${ }^{114}$ assessed various aspects of SLM manufactured parts with different scanning strategies. Their findings revealed that the scanning strategy could hinder the surface quality, mechanical properties, and grain direction in the SLM parts. Many scholars developed improvements in the grain structure and texture formation by SLM with various scanning techniques. It has been identified that texture control and an apparent grain growth mechanism can be achieved by changing the scanning strategy with a suitable selection of laser power and scanning speed..$^{105,115-118}$ Yang et al. ${ }^{23}$ proposed a fractal scanning path for selective laser sintering (SLS). His research showed that SLS samples generated along a fractal scanning path have enhanced physical efficiency. Compared with linear scanning, the grain sizes were more uniform and completely sintered using fractal scanning. Morgan et al. ${ }^{119}$ adopted a scanning strategy that creates laser re-melted components of stainless steel $(316 \mathrm{~L})$ with $<1 \%$ porosities while retaining the principle of rapid prototyping. Yasa et al. ${ }^{120}$ investigated the reasons for elevated edges in SLM. Different scan strategies and different hatching and contour parameters were evaluated to minimize the edge effect. In addition, for specific additive layer manufacturing components, fatigue loading is crucial and needs to be extensively investigated. Leuders et al. ${ }^{121}$ carried out a detailed microstructure and defected analysis to create deep mechanical property-microstructure relationships. According to the analysis, micron-sized pores have the greatest impact on fatigue strength, while residual stresses significantly impact fatigue crack growth. Mangour et al. ${ }^{122}$ studied the SLM process with different laser-scanning techniques to generate cylindrically shaped components from 316L stainless steel. It was found that the desired morphological and crystallographic textures of SLM-processed components characterized by either anisotropic or isotropic properties can be achieved by choosing the correct scanning strategy. Dimitrov et al. ${ }^{123}$ and Thijs et al. ${ }^{124}$ establish the effect of various scanning techniques and process parameters on the material efficiency of the SLM-manufactured Ti6Al4V parts. Their preliminary study reveals that the significant difference in the temperature distributions, temperature gradients, and cooling rates attributed to the building strategy used will result in substantial differences in the residual stresses and the microstructure of the components generated. Zhang and Shang ${ }^{125}$ analyzed two filling path generation algorithms, namely, the parallel reciprocating scan subarea and the parallel scan contour. This algorithm improved the efficiency of implementation, increased the effectiveness of scanning, and further improved the inner forming consistency of metal pieces. Microstructure determines mechanical properties, and some scholars have studied the influence of process parameters on the microstructure of SLM 316L. ${ }^{126,127}$ Investigation of the microstructure and shape of the tracks for process-parameter adjustments may provide valuable information for the creation of a comprehensive strategy for the production of SLM components with standardized microstructure and properties. ${ }^{29}$ Current research indicates that microstructural flaws inherent in AM processes must be managed and minimized to allow for full use of AM fabricated parts as structural components.

\section{Discussion}

Certainly, LAM has several advantages, including design versatility, print complex structures, ease of use, and product customization. However, some disadvantages and obstacles need to be investigated to advance technology development. The limitations on part size, mechanical 
properties, low manufacturing performance, lacking precision, warping, layer misalignment, mass production, and material limitations are all obstacles that require further research and development. ${ }^{128,129}$ Some of the constraints and challenges are described as follows.

\subsection{Mechanical Properties and Microstructure}

LAM fabricated part's microstructure and mechanical properties vary greatly depending on the build direction and other directions. ${ }^{109,130}$ Compared with numerical simulation and analysis, several essential data types, such as the microstructure and internal defects in LAM, are challenging to obtain by online monitoring. In the meantime, traditional equipment is easily disrupted by the surrounding environment. Therefore, more sophisticated equipment and technology, such as high-speed x-ray imaging and spatial resolution acoustic spectrum, must be used to obtain the required data.

\subsection{Deformation}

One of the biggest challenges in the LAM process is the deformation of the LAM fabricated part. Scanning patterns can have a greater effect on the structure and deformation of large components. A summary of different scanning patterns used in LBAM to analyze the impact of scanning patterns on deformation, stress, temperature, and mechanical properties is shown in Table 1. It has been seen in several studies that proper scanning patterns can significantly reduce deformation, but the parameters still need to be optimized. Parameters such as laser power, scanning

Table 1 Summary of scanning patterns.

\begin{tabular}{|c|c|c|c|}
\hline Scanning pattern & Category & Content & Publications \\
\hline Raster scanning & LMD & Thermal stress & 56 and 63 \\
\hline Raster scanning & LMD & Distortion & 51 \\
\hline Raster scanning & SLM & Microstructure and mechanical property & 103 and 122 \\
\hline Raster scanning & LMD & Deformation & 40 \\
\hline Axial and circumferential scan & LMD & Distortion and stress distribution & 52 \\
\hline Helix scan strategy & SLM & Deformation & 66 \\
\hline Fractal scanning & LMD & Distortion & 51 \\
\hline Fractal scanning & SLS & Temperature and stress analysis & 23 and 94 \\
\hline Spiral scanning & LMD & Distortion & 51 and 53 \\
\hline Spiral scanning & SLM & Stress and deformation & 11 \\
\hline Spiral scanning & LMD & Thermal analysis & $71-74$ and 95 \\
\hline Island scanning & SLM & Stress and deformation & 11,57, and 58 \\
\hline Island scanning & SLM & Grain structure and cracking behavior & 116 and 123 \\
\hline Unidirectional scanning & SLM & Residual stress and deformation & 25 and 26 \\
\hline Bidirectional scanning & SLM & Microstructure and mechanical property & $\begin{array}{c}104,106,117 \\
120,124, \text { and } 131\end{array}$ \\
\hline Parallel scanning & LMD & Distortion and residual stress & 64 \\
\hline Parallel scanning & SLM & Microstructure and mechanical property & 31 and 132 \\
\hline Parallel scanning & LMD & Cracking behavior & 113 \\
\hline Parallel scanning & LMD & Thermal and structural evaluation & $71-73,84$, and 91 \\
\hline
\end{tabular}


speed, and powder feed rate significantly impact deformation and part quality. ${ }^{33,87}$ In future studies, along with different scanning patterns, optimized process parameters should be implemented to minimize deformation and part quality issues. Some scholars used a laser displacement sensor (LDS) to predict the distortion in $\mathrm{AM}^{64,133}$ However, there was some irregularity in the data caused by a temporary power buildup on the LDS. Optimizing these issues in the sensor is also a great challenge for obtaining accurate data.

\subsection{Surface Quality}

Another issue encountered in LAM is the staircase effect or layering error in the fabricated pieces. Surface imperfections and defects such as staircase effects caused by layer-by-layer deposition methods, balling effects, and insufficient fusion result in a notably irregular microstructure. The surface roughness can significantly degrade the performance of laser additive-produced components, limiting their potential applications. ${ }^{134}$ Fatigue performance, wear and scratch resistance, and dimensional precision can be adversely affected by surface defects. Further research is needed to see how LAM's scanning strategy affects the produced parts' consistency, surface roughness, and material strength with new material powders.

\section{Conclusion}

LAM is a simple, low-cost, ${ }^{135,136}$ and long-lasting manufacturing technique, but concerns about component quality and reliability have hampered its widespread adoption. In LAM, many scholars have examined different scanning strategies to enhance the stability of the resultant product. In this paper, an overview of the research regarding the influence of scanning patterns on temperature field, deformation, and mechanical properties in LBAM is provided. Many scholars have done experiments and numerical simulations of different scanning patterns in the LAM process. Their results show that LAM's part quality issue can be minimized by choosing a proper scanning pattern and suitable process parameters such as laser power and scanning speed. This paper also addresses current issues and implementation directions of the scanning pattern in LBAM and should allow researchers to gain a clear view of the research status.

\section{Acknowledgments}

This research work was supported partially by the Science and Technology Innovation Fund of Dalian (Grant No. 2020JJ26GX040), the Fundamental Research Funds for the Central Universities (Grant No. DUT20JC19), the National Natural Science Foundation of China (Grant Nos. 51875082 and 51875075), and Major Science and Technology Innovation Funds of Shandong (Grant No. 2019JZZY010128).

\section{References}

1. H. Sahasrabudhe, S. Bose, and A. Bandyopadhyay, Laser-Based Additive Manufacturing Processes, 2nd ed., Washington State University, Pullman, Washington (2018).

2. O. Abdulhameed et al., "Additive manufacturing: challenges, trends, and applications," Adv. Mech. Eng. 11(2), 168781401882288 (2019).

3. D. Chen et al., "Direct digital manufacturing: definition, evolution, and sustainability implications," J. Clean. Prod. 107, 615-625 (2015).

4. J. Du et al., "Numerical analysis of fused-coating metal additive manufacturing," Int. J. Therm. Sci. 114, 342-351 (2017).

5. J. Song et al., "Research progress of laser cladding forming technology," Jixie Gongcheng Xuebao/J. Mech. Eng. 46(14), 29-39 (2010).

6. A. Uriondo, M. Esperon-Miguez, and S. Perinpanayagam, "The present and future of additive manufacturing in the aerospace sector: a review of important aspects," Proc. Inst. Mech. Eng. Part G 229(11), 2132-2147 (2015). 
7. I. Yadroitsev, P. Krakhmalev, and I. Yadroitsava, "Selective laser melting of Ti6Al4V alloy for biomedical applications: temperature monitoring and microstructural evolution," J. Alloys Compd. 583, 404-409 (2014).

8. W. Liu and J. N. DuPont, "Fabrication of functionally graded TiC/Ti composites by laser engineered net shaping," Scr. Mater. 48(9), 1337-1342 (2003).

9. W. E. Frazier, "Metal additive manufacturing: a review," J. Mater. Eng. Perform. 23(6), 1917-1928 (2014).

10. M. L. Griffith et al., "Understanding the microstructure and properties of components fabricated by laser engineered net shaping (LENS)," MRS Online Proc. Libr. 625(1), 9 (2000).

11. B. Cheng, S. Shrestha, and K. Chou, "Stress and deformation evaluations of scanning strategy effect in selective laser melting," Addit. Manuf. 12, 240-251 (2016).

12. S. A. Khairallah and A. Anderson, "Mesoscopic simulation model of selective laser melting of stainless steel powder," J. Mater. Process. Technol. 214(11), 2627-2636 (2014).

13. E. Yasa et al., "Charpy impact testing of metallic selective laser melting parts," Virtual Phys. Prototyp. 5(2), 89-98 (2010).

14. Z. J. Tang et al., "A review on in situ monitoring technology for directed energy deposition of metals," Int. J. Adv. Manuf. Technol. 108(11-12), 3437-3463 (2020).

15. X. X. Yao et al., "Controlling the solidification process parameters of direct energy deposition additive manufacturing considering laser and powder properties," Comput. Mater. Sci. 182, 109788 (2020).

16. D. De Baere et al., "Spectroscopic monitoring and melt pool temperature estimation during the laser metal deposition process," J. Laser Appl. 28(2), 022303 (2016).

17. C. Valente, T. Morgado, and N. Sharma, "LASER cladding-a post processing technique for coating, repair and re-manufacturing," in Materials Forming, Machining and Post Processing, Materials Forming, Machining and Tribology, K. Gupta, Ed., Springer, Cham, pp. 231-249 (2020).

18. J. C. Heigel, P. Michaleris, and E. W. Reutzel, "Thermo-mechanical model development and validation of directed energy deposition additive manufacturing of Ti-6Al-4V," Addit. Manuf. 5, 9-19 (2015).

19. H. Wang et al., "Review on adaptive control of laser-directed energy deposition," Opt. Eng. 59(7), 070901 (2020).

20. J. Cao, M. A. Gharghouri, and P. Nash, "Finite-element analysis and experimental validation of thermal residual stress and distortion in electron beam additive manufactured Ti-6Al-4V build plates," J. Mater. Process. Technol. 237, 409-419 (2016).

21. Z. Yan et al., "Review on thermal analysis in laser-based additive manufacturing," Opt. Laser Technol. 106, 427-441 (2018).

22. Z. Zhang et al., "Numerical studies of residual states and scaling effects in laser-directed energy deposition additive manufacturing," Int. J. Adv. Manuf. Technol. 108(4), 12331247 (2020).

23. J. Yang et al., "Fractal scanning path generation and control system for selective laser sintering (SLS)," Int. J. Mach. Tools Manuf. 43(3), 293-300 (2003).

24. S. Son, S. Kim, and K. H. Lee, "Path planning of multi-patched freeform surfaces for laser scanning," Int. J. Adv. Manuf. Technol. 22(5-6), 424-435 (2003).

25. L. Parry, I. A. Ashcroft, and R. D. Wildman, "Understanding the effect of laser scan strategy on residual stress in selective laser melting through thermo-mechanical simulation," Addit. Manuf. 12, 1-15 (2016).

26. D. Ramos, F. Belblidia, and J. Sienz, "New scanning strategy to reduce warpage in additive manufacturing," Addit. Manuf. 28, 554-564 (2019).

27. D. Zhao and W. Guo, "Shape and performance controlled advanced design for additive manufacturing: a review of slicing and path planning," J. Manuf. Sci. Eng. Trans. ASME 142(1), 1-23 (2020).

28. I. Yadroitsev et al., "Single track formation in selective laser melting of metal powders," J. Mater. Process. Technol. 210(12), 1624-1631 (2010).

29. I. Yadroitsev et al., "Energy input effect on morphology and microstructure of selective laser melting single track from metallic powder," J. Mater. Process. Technol. 213(4), 606613 (2013). 
30. I. Yadroitsev et al., "Factor analysis of selective laser melting process parameters and geometrical characteristics of synthesized single tracks," Rapid Prototyp. J. 18(3), 201-208 (2012).

31. Y. A. Jin et al., "A parallel-based path generation method for fused deposition modeling," Int. J. Adv. Manuf. Technol. 77(5-8), 927-937 (2015).

32. M. Boissier, G. Allaire, and C. Tournier, "Additive manufacturing scanning paths optimization using shape optimization tools," Struct. Multidiscip. Optim. 61(6), 2437-2466 (2020).

33. M. Carraturo et al., "Numerical evaluation of advanced laser control strategies influence on residual stresses for laser powder bed fusion systems," Integr. Mater. Manuf. Innov. 9(4), 435-445 (2020).

34. W. Zhang et al., "Consecutive sub-sector scan mode with adjustable scan lengths for selective laser melting technology," Int. J. Adv. Manuf. Technol. 41(7-8), 706-713 (2009).

35. J. Jhabvala et al., "On the effect of scanning strategies in the selective laser melting process," Virtual Phys. Prototyp. 5(2), 99-109 (2010).

36. D. Shishi et al., "Planning of area partition scanning path and its effect on residual stress of SLM molding parts," Chin. J. Lasers 43(12), 1202003 (2016).

37. J. Zhao et al., "Research on algorithm of optimized sub-regional scanning path generation for RPM," in Proc. IEEE Int. Conf. Mechatron. Autom., pp. 587-592 (2007).

38. K.-H. Choi et al., "Study on path generation and control based on dual laser in solid freefrom fabrication system," in SICE-ICASE Int. Joint Conf., pp. 3682-3687 (2006).

39. J. Fessler et al., "Laser deposition of metals for shape deposition manufacturing," in Solid Free. Fabr. Symp. Proc., University of Texas Austin, pp. 117-124 (1996).

40. M. Gao et al., "The effect of deposition patterns on the deformation of substrates during direct laser fabrication," J. Eng. Mater. Technol. ASME 135(3), 1-6 (2013).

41. F. B. Prinz et al., "Processing, thermal and mechanical issues in shape deposition manufacturing," in Solid Free. Fabr. Symp. Proc., pp. 118-129 (1995).

42. C. H. Amon and R. Merz, "Shape deposition manufacturing with microcasting: processing, thermal and mechanical issues," J. Manuf. Sci. Eng. 120(3), 656-665 (1998).

43. S. Zou et al., "Numerical analysis of the effect of the scan strategy on the residual stress in the multi-laser selective laser melting," Results Phys. 16, 103005 (2020).

44. M. Biegler et al., "Finite element analysis of in-situ distortion and bulging for an arbitrarily curved additive manufacturing directed energy deposition geometry," Addit. Manuf. 24, 264-272 (2018).

45. M. Biegler, B. Graf, and M. Rethmeier, "In-situ distortions in LMD additive manufacturing walls can be measured with digital image correlation and predicted using numerical simulations," Addit. Manuf. 20, 101-110 (2018).

46. Z. Nie et al., "Experimental study and modeling of H13 steel deposition using laser hot-wire additive manufacturing," J. Mater. Process. Technol. 235, 171-186 (2016).

47. Z. Chen, H. Ye, and H. Xu, "Distortion control in a wire-fed electron-beam thin-walled Ti-6Al-4V freeform," J. Mater. Process. Technol. 258, 286-295 (2018).

48. Q. Chen et al., "An inherent strain based multiscale modeling framework for simulating part-scale residual deformation for direct metal laser sintering," Addit. Manuf. 28, 406-418 (2019).

49. P. Foteinopoulos et al., "Development of a simulation approach for laser powder bed fusion based on scanning strategy selection," Int. J. Adv. Manuf. Technol. 108(9-10), 3085-3100 (2020).

50. S. Afazov et al., "Distortion prediction and compensation in selective laser melting," Addit. Manuf. 17, 15-22 (2017).

51. J. Yu et al., "Influence of laser deposition patterns on part distortion, interior quality and mechanical properties by laser solid forming (LSF)," Mater. Sci. Eng. A 528(3), 1094 1104 (2011).

52. S. Safdar et al., "Finite element simulation of laser tube bending: effect of scanning schemes on bending angle, distortions and stress distribution," Opt. Laser Technol. 39(6), 1101-1110 (2007). 
53. K. Dai and L. Shaw, "Distortion minimization of laser-processed components through control of laser scanning patterns," Rapid Prototyp. J. 8(5), 270-276 (2002).

54. K. Dai and L. Shaw, "Parametric studies of multi-material laser densification," Mater. Sci. Eng. A 430(1-2), 221-229 (2006).

55. L. Papadakis et al., "Numerical computation of component shape distortion manufactured by selective laser melting," Procedia CIRP 18, 90-95 (2014).

56. A. H. Nickel, D. M. Barnett, and F. B. Prinz, "Thermal stresses and deposition patterns in layered manufacturing," Mater. Sci. Eng. A 317(1-2), 59-64 (2001).

57. M. F. Zaeh and G. Branner, "Investigations on residual stresses and deformations in selective laser melting," Prod. Eng. 4(1), 35-45 (2010).

58. H. Yan et al., "Stress and deformation evaluation of the subarea scanning effect in direct laser-deposited Ti-6Al-4V," Int. J. Adv. Manuf. Technol. 97(1-4), 915-926 (2018).

59. D. Hagedorn-Hansen et al., "The effects of selective laser melting scan strategies on deviation of hybrid parts," South African J. Ind. Eng. 28, 200-212 (2017).

60. C. Li et al., "Fast prediction and validation of part distortion in selective laser melting," Procedia Manuf. 1, 355-365 (2015).

61. X. Lu et al., "Residual stress and distortion of rectangular and S-shaped Ti-6Al-4V parts by directed energy deposition: modelling and experimental calibration," Addit. Manuf. 26, 166-179 (2019).

62. N. W. Klingbeil et al., "Residual stress-induced warping in direct metal solid freeform fabrication," Int. J. Mech. Sci. 44(1), 57-77 (2002).

63. A. Nickel, "Analysis of thermal stresses in shape deposition manufacturing of metal parts C" (1999).

64. E. R. Denlinger et al., "Effect of inter-layer dwell time on distortion and residual stress in additive manufacturing of titanium and nickel alloys," J. Mater. Process. Technol. 215, 123-131 (2015).

65. J. P. Kruth et al., "Selective laser melting of iron-based powder," J. Mater. Process. Technol. 149(1-3), 616-622 (2004).

66. B. Qian et al., "The helix scan strategy applied to the selective laser melting," Int. J. Adv. Manuf. Technol. 63(5-8), 631-640 (2012).

67. J. Song et al., "Role of scanning strategy on residual stress distribution in Ti-6Al-4V alloy prepared by selective laser melting," Optik 170, 342-352 (2018).

68. Z. Yan et al., "Effect of thermal characteristics on distortion in laser cladding of AISI 316L," J. Manuf. Process. 44, 309-318 (2019).

69. J. C. Heigel, P. Michaleris, and T. A. Palmer, "In situ monitoring and characterization of distortion during laser cladding of Inconel ${ }^{\circledR}$ 625," J. Mater. Process. Technol. 220, 135145 (2015).

70. M. L. Griffith et al., "Understanding thermal behavior in the LENS process," Mater. Des. 20(2-3), 107-113 (1999).

71. X. Gong, J. Luo, and D. Hu, "Effect of scanning path on temperature field in laser cladding," IOP Conf Ser. Mater. Sci. Eng. 207(1), 012039 (2017).

72. E. Foroozmehr and R. Kovacevic, "Effect of path planning on the laser powder deposition process: thermal and structural evaluation," Int. J. Adv. Manuf. Technol. 51(5-8), 659-669 (2010).

73. T. Asikainen, M. Ritala, and L. Markku, "Atomic layer deposition growth of zirconium doped $\mathrm{In}_{2} \mathrm{O}_{3}$ films," Thin Solid Films 440, 152-154 (2003).

74. S. Ghosh and J. Choi, "Deposition pattern based thermal stresses in single-layer laser aided direct material deposition process," J. Manuf. Sci. Eng. Trans. ASME 129(2), 319-332 (2007).

75. R. B. Patil and V. Yadava, "Finite element analysis of temperature distribution in single metallic powder layer during metal laser sintering," Int. J. Mach. Tools Manuf. 47(7-8), 1069-1080 (2007).

76. M. Matsumoto et al., "Finite element analysis of single layer forming on metallic powder bed in rapid prototyping by selective laser processing," Int. J. Mach. Tools Manuf. 42(1), 61-67 (2002). 
77. S. Kolossov et al., "3D FE simulation for temperature evolution in the selective laser sintering process," Int. J. Mach. Tools Manuf. 44(2-3), 117-123 (2004).

78. G. Bugeda, M. Cervera, and G. Lombera, "Numerical prediction of temperature and density distributions in selective laser sintering processes," Rapid Prototyp. J. 5(1), 21-26 (1999).

79. B. Q. Chen, M. Hashemzadeh, and C. G. Soares, "Numerical and experimental studies on temperature and distortion patterns in butt-welded plates," Int. J. Adv. Manuf. Technol. 72(5-8), 1121-1131 (2014).

80. A. Hussein et al., "Finite element simulation of the temperature and stress fields in single layers built without-support in selective laser melting," Mater. Des. 52, 638-647 (2013).

81. K. Antony, N. Arivazhagan, and K. Senthilkumaran, "Numerical and experimental investigations on laser melting of stainless steel 316L metal powders," J. Manuf. Process. 16(3), 345-355 (2014).

82. E. R. Denlinger and P. Michaleris, "Effect of stress relaxation on distortion in additive manufacturing process modeling," Addit. Manuf. 12, 51-59 (2016).

83. N. Patil, D. Pal, and B. Stucker, "A new finite element solver using numerical Eigen modes for fast simulation of additive manufacturing processes," in 24th Int. SFF Symp.—An Addit. Manuf. Conf., pp. 535-548 (2013).

84. K. Dai and L. Shaw, "Thermal and stress modeling of multi-material laser processing," Acta Mater. 49(20), 4171-4181 (2001).

85. ANSYS Inc., "ANSYS theory reference - release 5.6," Theory Reference, pp. 1-1286, http:// research.me.udel.edu/ lwang/teaching/MEx81/ansys56manual.pdf (November 1999).

86. P. Kohnk, "Ansys theory reference for the mechanical APDL and mechanical applications," Ansys 3304, 724-746 (2009).

87. P. Bian et al., "Influence of laser power and scanning strategy on residual stress distribution in additively manufactured 316L steel," Opt. Laser Technol. 132, 106477 (2020).

88. A. M. de Deus and J. Mazumder, "Two-dimensional thermo-mechanical finite element model for laser cladding," ICALEO 1996, B174-B183 (2018).

89. F.-J. Kahlen and A. Kar, "Residual stresses in laser-deposited metal parts," J. Laser Appl. 13(2), 60-69 (2001).

90. I. A. Roberts et al., "A three-dimensional finite element analysis of the temperature field during laser melting of metal powders in additive layer manufacturing," Int. J. Mach. Tools Manuf. 49(12-13), 916-923 (2009).

91. X. U. Haiyan et al., "Study on quality prediction and path selection of 316L laser cladding," Laser Technol. 42(1), 3-9 (2018).

92. T. Mukherjee, W. Zhang, and T. DebRoy, "An improved prediction of residual stresses and distortion in additive manufacturing," Comput. Mater. Sci. 126, 360-372 (2017).

93. M. Alimardani, E. Toyserkani, and J. P. Huissoon, "A 3D dynamic numerical approach for temperature and thermal stress distributions in multilayer laser solid freeform fabrication process," Opt. Lasers Eng. 45(12), 1115-1130 (2007).

94. L. Ma and H. Bin, "Temperature and stress analysis and simulation in fractal scanningbased laser sintering," Int. J. Adv. Manuf. Technol. 34(9-10), 898-903 (2007).

95. K. Ren et al., "Thermal analyses for optimal scanning pattern evaluation in laser aided additive manufacturing," J. Mater. Process. Technol. 271, 178-188 (2019).

96. K. Ren et al., "Thermal field prediction for laser scanning paths in laser aided additive manufacturing by physics-based machine learning," Comput. Methods Appl. Mech. Eng. 362, 112734 (2020).

97. Z. Luo and Y. Zhao, "A survey of finite element analysis of temperature and thermal stress fields in powder bed fusion additive manufacturing," Addit. Manuf. 21, 318-332 (2018).

98. O. Zinovieva, A. Zinoviev, and V. Ploshikhin, "Three-dimensional modeling of the microstructure evolution during metal additive manufacturing," Comput. Mater. Sci. 141, 207220 (2018).

99. Z. Zhang et al., "Numerical methods for microstructural evolutions in laser additive manufacturing," Comput. Math. Appl. 78(7), 2296-2307 (2019). 
100. J. Z. Su et al., "Microstructural morphology and evolution of austenite stainless steel deposited using pulsed laser and wire," Int. J. Adv. Manuf. Technol. 93(9-12), 33573370 (2017).

101. P. Ge et al., "An integrated modeling of process-structure-property relationship in laser additive manufacturing of duplex titanium alloy," Int. J. Therm. Sci. 140, 329-343 (2019).

102. W. Yan et al., "Modeling process-structure-property relationships for additive manufacturing," Front. Mech. Eng. 13(4), 482-492 (2018).

103. F. Liu et al., "The effect of laser scanning path on microstructures and mechanical properties of laser solid formed nickel-base superalloy Inconel 718," J. Alloys Compd. 509(13), 4505-4509 (2011).

104. A. Kudzal et al., "Effect of scan pattern on the microstructure and mechanical properties of Powder Bed Fusion additive manufactured 17-4 stainless steel," Mater. Des. 133, 205-215 (2017).

105. H. Y. Wan et al., "Effect of scanning strategy on mechanical properties of selective laser melted Inconel 718," Mater. Sci. Eng. A 753, 42-48 (2019).

106. C. Y. Liu et al., "Effect of scanning strategy on microstructure and mechanical properties of selective laser melted reduced activation ferritic/martensitic steel," Mater. Sci. Eng. A 766, 138364 (2019).

107. R. Rashid et al., "Effect of scan strategy on density and metallurgical properties of 17-4PH parts printed by selective laser melting (SLM)," J. Mater. Process. Technol. 249, 502-511 (2017).

108. F. Liu et al., "Microstructure and residual stress of laser rapid formed Inconel 718 nickelbase superalloy," Opt. Laser Technol. 43(1), 208-213 (2011).

109. B. Vrancken et al., "Heat treatment of Ti6Al4V produced by selective laser melting: microstructure and mechanical properties," J. Alloys Compd. 541, 177-185 (2012).

110. W. Woo et al., "Effect of interlayers and scanning strategies on through-thickness residual stress distributions in additive manufactured ferritic-austenitic steel structure," Mater. Sci. Eng., A 744, 618-629 (2019).

111. H. Ali, H. Ghadbeigi, and K. Mumtaz, "Effect of scanning strategies on residual stress and mechanical properties of selective laser melted Ti6Al4V," Mater. Sci. Eng. A 712, 175-187 (2018).

112. I. Yadroitsev, P. Krakhmalev, and I. Yadroitsava, "Hierarchical design principles of selective laser melting for high quality metallic objects," Addit. Manuf. 7, 45-56 (2015).

113. L. Ri-Sheng, S. Shao-Ni, and L. Zi-Sheng, "The influence of scanning methods on the cracking failure of thin-wall metal parts fabricated by laser direct deposition shaping," Eng. Fail. Anal. 59, 269-278 (2016).

114. J. P. Kruth et al., "Part and material properties in selective laser melting of metals," in 16th Int. Symp. Electromach., pp. 3-14 (2010).

115. H. L. Wei, J. Mazumder, and T. DebRoy, "Evolution of solidification texture during additive manufacturing," Sci. Rep. 5, 16446 (2015).

116. L. N. Carter et al., "The influence of the laser scan strategy on grain structure and cracking behaviour in SLM powder-bed fabricated nickel superalloy," J. Alloys Compd. 615, 338347 (2014).

117. S. H. Sun, K. Hagihara, and T. Nakano, "Effect of scanning strategy on texture formation in Ni-25 at.\%Mo alloys fabricated by selective laser melting," Mater. Des. 140, 307-316 (2018).

118. N. Raghavan et al., "Localized melt-scan strategy for site specific control of grain size and primary dendrite arm spacing in electron beam additive manufacturing," Acta Mater. 140, 375-387 (2017).

119. R. H. Morgan et al., "High density net shape components by direct laser re-melting of single-phase powders," J. Mater. Sci. 37(15), 3093-3100 (2002).

120. E. Yasa et al., "Investigation on occurrence of elevated edges in selective laser melting," Asp. Gen. La Planif. Tribut. En Venez. 2009(75), 31-47 (2009).

121. S. Leuders et al., "On the mechanical behaviour of titanium alloy TiAl6V4 manufactured by selective laser melting: fatigue resistance and crack growth performance," Int. J. Fatigue 48, 300-307 (2013). 
122. B. AlMangour, D. Grzesiak, and J. M. Yang, "Scanning strategies for texture and anisotropy tailoring during selective laser melting of TiC/316L stainless steel nanocomposites," J. Alloys Compd. 728, 424-435 (2017).

123. D. Dimitrov et al., "On the impact of different system strategies on the material performance of selective laser melting- manufactured TI6AL4V components," South African J. Ind. Eng. 27(3Speciallssue), 184-191 (2016).

124. L. Thijs et al., "A study of the microstructural evolution during selective laser melting of Ti-6Al-4V," Acta Mater. 58(9), 3303-3312 (2010).

125. K. Zhang and X. Shang, "Data processing of laser metal deposition shaping technology: Part III. Filling scanning path research," Appl. Mech. Mater. 58-60, 2644-2648 (2011).

126. J. Liu et al., "Effect of scanning speed on the microstructure and mechanical behavior of 316L stainless steel fabricated by selective laser melting," Mater. Des. 186, 108355 (2020).

127. R. Casati, J. Lemke, and M. Vedani, "Microstructure and fracture behavior of 316L austenitic stainless steel produced by selective laser melting," J. Mater. Sci. Technol. 32(8), 738-744 (2016).

128. J. P. J. De Jong and E. De Bruijn, "Innovation lessons from 3-D printing," MIT Sloan Manage. Rev. 52(2), 43-52 (2013).

129. L. Chen et al., "The research status and development trend of additive manufacturing technology," Int. J. Adv. Manuf. Technol. 89(9), 3651-3660 (2017).

130. S. H. Ahn et al., "Anisotropic material properties of fused deposition modeling ABS," Rapid Prototyp. J. 8(4), 248-257 (2002).

131. H. Y. Wan et al., "Effect of scanning strategy on grain structure and crystallographic texture of Inconel 718 processed by selective laser melting," J. Mater. Sci. Technol. 34(10), 17991804 (2018).

132. R. Stamp et al., "The development of a scanning strategy for the manufacture of porous biomaterials by selective laser melting," J. Mater. Sci. - Mater. Med. 20(9), 1839-1848 (2009).

133. K. Masubuchi, "Prediction and control of residual stresses and distortion in welded structures osaka university knowledge archive," Trans. JWRI 25(2), 53-67 (1996).

134. E. Maleki et al., "Surface post-treatments for metal additive manufacturing: progress, challenges, and opportunities," Addit. Manuf. 37, 101619 (2021).

135. D. S. Thomas and S. W. Gilbert, "Costs and cost effectiveness of additive manufacturing: a literature review and discussion," Addit. Manuf. Costs, Cost Eff. Ind. Econ., pp. 1-96 (2015).

136. E. Atzeni and A. Salmi, "Economics of additive manufacturing for end-usable metal parts," Int. J. Adv. Manuf. Technol. 62(9-12), 1147-1155 (2012).

Wei-Wei Liu is an associate professor at the School of Mechanical Engineering, Dalian University of Technology. His research focuses on AM, LDED, machine vision, and intelligent control systems.

Biographies of the other authors are not available. 